\title{
Synovial Sarcoma: A Clinicopathological Study with Emphasis on Rare Sites
}

\author{
Imtiaz Ahmed, Divya Shelly*, K V Vinu Balraam, Kriti Agarwal and Reena Bharadwaj \\ Department of Pathology Armed Forces Medical College, Pune. India
}

\begin{abstract}
Background: Synovial sarcoma is an infrequently diagnosed soft tissue sarcoma of the younger population with a male preponderance. Although the disease tends to home itself in the extremities particularly around the joints in the vast majority of cases, it can also occur in other surprising locations which warrant careful and meticulous histopathological examination accompanied by a judicious use of the immunohistochemical panel.

Methods: Search of the pathology database was done to identify cases of synovial sarcomas in rare sites.

Result: seven cases of synovial sarcoma were identified, three of which were at extremely uncommon sites rendering the diagnosis challenging and intriguing.

Conclusion: Differential diagnosis of synovial sarcoma should always be kept in mind in a young patient with a mass lesion of short duration, not only at the extremities but also involving other rarer locations like the lungs, heart and kidney.
\end{abstract}

Keywords: Clinicopathological Study; Heart; Kidney; Lung; Synovial Sarcoma.

\section{Introduction}

Synovial sarcoma is a clinically and histomorphologically distinct entity in the soft tissue sarcoma family and classified under 'tumour with uncertain differentiation' in the '2013 World Health Organization (WHO) Classification of Tumors of Soft Tissue and Bone'. ${ }^{[1]}$ The description of synovial sarcoma dates back to 1865 when Simon described a pedunculated soft tissue tumour with a smooth surface in the right knee of a 46-year-old man. ${ }^{[2]}$ But the term 'synovial sarcoma' was only coined in 1934 in France thinking it to originate from the synovial membrane of joints. ${ }^{[2]}$ However, it was only later acknowledged through electron microscopy, cytogenetic and molecular studies that the origin of synovial sarcoma is not from the synovial membrane, but rather from pluripotent mesenchymal stem cells which can differentiate both into epithelial and mesenchymal elements. ${ }^{[3,4]}$ It was further supported by the fact that both the epithelial and spindle cell component harboured the same genetic defect. ${ }^{[4]}$ Initially, biphasic synovial sarcomas were described more in literature and were identified as spindle cell tumours demonstrating an epithelial component, which was deemed necessary to type a tumour as synovial sarcoma. ${ }^{[5]}$ Later, the monophasic and poorly differentiated synovial sarcoma were described with similar cytogenetic abnormalities. ${ }^{[4]}$

Synovial sarcoma accounts for $8 \%$ to $10 \%$ of all human malignancies, with $90 \%$ of them affecting the extremities.
Of this $30 \%$ occur around the knee joint. ${ }^{[2,3]}$ Apart from knee joint it is known to occur at several other locations such as head and neck, lungs, heart, kidney, mediastinum and retroperitoneum to name a few. ${ }^{[2,4]}$ It is a malignancy of the young with the majority of the cases occurring at a mean age of 26 years. ${ }^{[2]}$

In our study, we present seven cases of synovial sarcoma, out of which three occurred at rare locations of the body. While the diagnosis was straightforward in extremities, the location of tumour at rare sites entailed the use of immunohistochemistry (IHC) to nail the correct diagnosis.

\section{Material and Methods}

A search of the pathology database of our institution identified seven cases of synovial sarcoma diagnosed between January 2017 and December 2017. The clinical records of all the patients were documented. HaematoxylinEosin stained slides and immunohistochemistry (IHC) findings were reviewed. A compilation was made for all the cases keeping in mind the age, sex, location, histomorphological and IHC features of the tumour.

\section{Results}

\section{Clinical Features}

Clinicopathological features of the seven cases are summarized in Table 1. The patient's age ranged from 29 to 58 years. All the patients were males. Out of seven cases, three had presented with tumour at rare sites, viz, left lung 
(left upper lobe), left kidney and right atrium and none of the cases had suspected clinical diagnosis of sarcoma.

Of the the three cases, two cases (left lung mass and right atrial mass) were in-patient cases whereas one case (left renal mass) was referred for a second opinion. For the inpatient cases, core needle biopsy and resection specimens were available for analysis.

Clinical presentation of Case $\mathbf{1}$ is worth noting and especially intriguing. This was a 25 -year-old male who came to medical attention with symptoms of cough, dyspnea on exertion, weight loss and hemoptysis off and on for last 6 months. He was clinically diagnosed with sputum-negative pulmonary tuberculosis and was started on anti-tubercular drugs. There was no relief in his symptoms even after 3 months of starting ATT and he subsequently was referred to our centre. Detailed imaging studies were done at our centre which detected a right mediastinal mass. Positron emission tomography(PET) scan was suggestive of right atrial mass. He was taken up for thoracotomy for biopsy and if possible removal of the mass but the surgery had to be abandoned due to cardiac complications. He succumbed to his illness and a detailed autopsy was carried out. It was only during the autopsy that a large mass occupying the whole atrium was noted which was infiltrating the right ventricular cavity. The mass was solid, greyish white and was also infiltrating the major vessels also (Figure 1). Case 2 was of a 58-year-old reformed smoker who presented with dyspnea and exertion along with left sided pleural effusion. The PET-CT imaging scan revealed $13 \times 10 \times 8 \mathrm{~cm}$ mass in the left upper lobe of the lung with a maximum SUV of 2.94, along with mild pleural effusion (Figure 2). Case 3 was of a 32-year-old male patient with right kidney mass who underwent radical nephrectomy (Figure 3). Rest of the cases presented with soft tissue tumours of extremities.

\section{Microscopic and Immunohistochemical Features}

All cases were diagnosed as monophasic synovial sarcoma. The predominant pattern was of a spindle cell tumour with tumour cells arranged in sheets and fascicular arrangement. Nuclear pleomorphism was moderate. Mitotic activity was variable from $3-4 / 10 \mathrm{HPF}$ to $7-8 / 10$ HPF. The prominent hemangiopericytomatous pattern of the blood vessels within the tumour stroma was noted in 2/7 cases. Areas of myxoid changes were also seen in $1 / 7$ cases. Areas of thick collagen bundles were noted in 4/7 cases. Case 2 showed areas of necrosis. Hence, a diverse histomorphological characteristic of the tumour was noted in different cases which reveal the whole histomorphological spectrum of this tumour. The diagnosis of synovial sarcoma was complemented by the IHC panel put in each of the cases. The panel comprised of CK, EMA, SMA, Desmin, S100P, CD34, CD99, BCL2, calponin and TLE-1. Additional immunohistochemistry panel was put in those cases where another specific malignancy was to be ruled out like CD10 for renal carcinoma and TTF- 1 for ruling out lung primary. All of the tumours consistently showed diffuse positivity for vimentin, BCL2 and TLE-1, which is a novel marker for synovial sarcoma. ${ }^{[6,7]}$ Keratin positivity in spindle component was seen in 5/7 (71\%) cases. Immunohistochemical features are summarized in Table 2.

TABLE 1: Clinicopathological features of the tumours

\begin{tabular}{|l|l|l|l|l|}
\hline Case No & Age/Sex & Organ & Specimen type & Size \\
\hline 1 & $29 / \mathrm{M}$ & Right atrium & Heart (autopsy) & $11 \mathrm{~cm}$ \\
\hline 2 & $58 / \mathrm{M}$ & Lung mass - Left upper lobe & Trucut biopsy and main specimen & $21 \mathrm{~cm}$ \\
\hline 3 & $32 / \mathrm{M}$ & Left kidney & Blocks for review & $12 \mathrm{~cm}$ \\
\hline 4. & $27 / \mathrm{F}$ & Left forearm & Trucut biopsy & $6 \mathrm{~cm}$ \\
\hline 5. & $25 / \mathrm{M}$ & Left thigh & Trucut biopsy & $6 \mathrm{~cm}$ \\
\hline 6. & $60 / \mathrm{M}$ & Right thigh & Block for review & Referral case \\
\hline 7. & 22/M & Right leg & Below knee amputation & $16 \mathrm{~cm}$ \\
\hline
\end{tabular}

TABLE 2: Immunohistochemical features of the tumours.

\begin{tabular}{|l|l|l|}
\hline Case No. & Histological diagnosis & Immunohistochemistry \\
\hline 1. & Monophasic synovial sarcoma & $\begin{array}{l}\text { Positive for vimentin, CD99, calponin, BCL2 and TLE-1. Negative for } \\
\text { CK, EMA, SMA, CD34, S100p }\end{array}$ \\
\hline 2. & Monophasic synovial sarcoma & $\begin{array}{l}\text { Positive for vimentin, EMA, CK, calponin, BCL2 and TLE-1. Negative } \\
\text { for SMA, Desmin, S100p, CD31, CD34, p63 and TTF-1. }\end{array}$ \\
\hline 3. & Monophasic synovial sarcoma & $\begin{array}{l}\text { Positive for vimentin, CD99, CK, EMA, BCL2 and TLE-1. Negative for } \\
\text { CD10, SMA, Desmin and CD34. }\end{array}$ \\
\hline
\end{tabular}




\begin{tabular}{|c|c|c|}
\hline Case No. & Histological diagnosis & Immunohistochemistry \\
\hline 4. & Monophasic synovial sarcoma & $\begin{array}{l}\text { Positive for Vimentin, CD99, BCL2 and TLE-1. Negative for CK, EMA, } \\
\text { SMA, desmin, CD34, S100p. }\end{array}$ \\
\hline 5. & Monophasic synovial sarcoma & $\begin{array}{l}\text { Positive for Vimentin, CD99, BCL2, EMA and TLE-1. Negative for } \\
\text { SMA, CD34, S100p. }\end{array}$ \\
\hline 6. & Monophasic synovial sarcoma & $\begin{array}{l}\text { Positive for Vimentin, BCL2, CK, EMA and TLE-1. Negative for SMA, } \\
\text { Desmin and CD34. }\end{array}$ \\
\hline 7. & Monophasic synovial sarcoma & $\begin{array}{l}\text { Positive for Vimentin, CD99, EMA, BCL2 and TLE-1. Negative for } \\
\text { EMA, S100p, SMA, CD34. }\end{array}$ \\
\hline
\end{tabular}

*CK: Pan-cytokeratin, EMA: Epithelial membrane antigen, SMA: smooth muscle antigen, BCL2: B-cell lymphoma 2, S100p-S100 protein, TLE-1: Transducinlike enhancer protein 1, TTF-1: Thyroid transcription factor-1, CD: Cluster of designation.

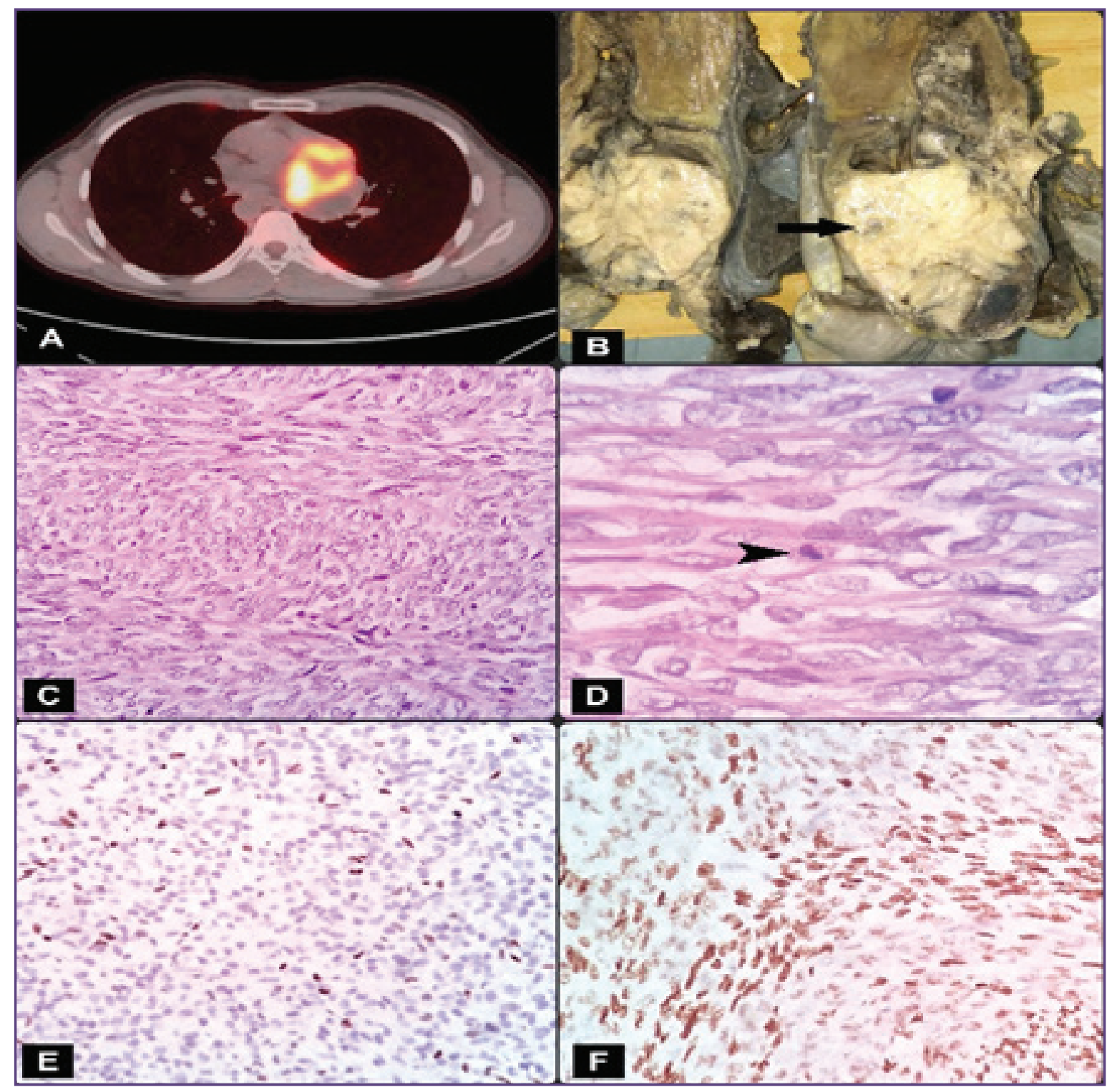

Fig. 1(Case 1): A,PET scan revealing a FDG avid right atrial mass of Case 1.B, Mass seen during autopsy occupying the whole of the right atrium. C, Low magnification revealed a monophasic spindle cell tumor with predominant herringbone pattern (H\&E stain, 100X). D, Mitotic activity was noted (arrow head) (H\&E stain, 400X). E, Mib-1 labelling index was 5-10\%. F,Tumour cells showed diffuse positivity with TLE-1 immunostains. 


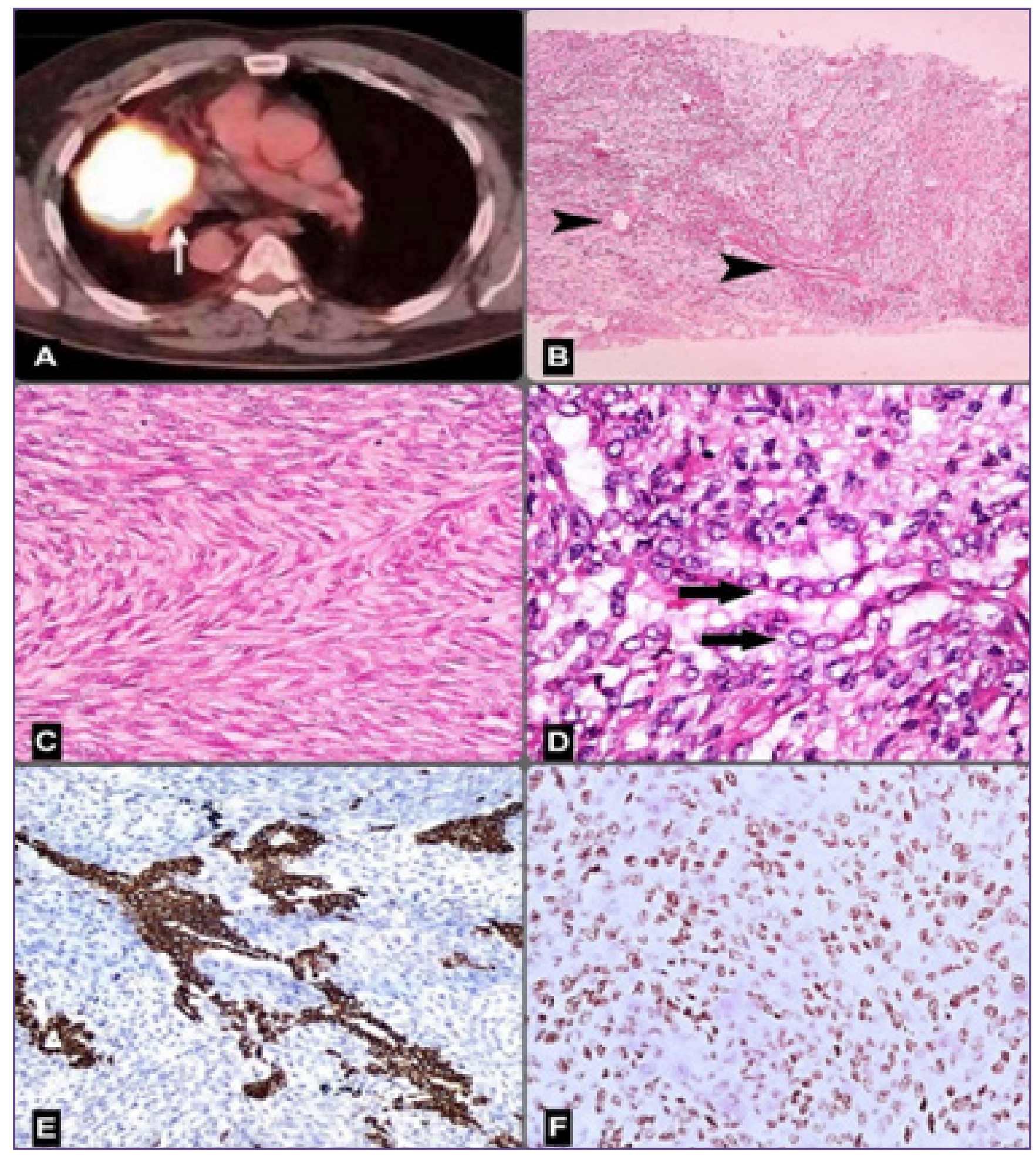

Fig. 2: (Case 2) A,PET scan revealing a a large $13 \times 10 \mathrm{~cm}$ mass in the left upper lobe of lung in case 2. B, Initial trucut biopsy of the specimen revealing a spindle cell tumor with hemangiopericytomatous blood vessels (arrow heads) (H\&E stain, 40X) C, Low magnification revealed a monophasic spindle cell tumor with predominant herringbone pattern (H\&E stain, 100X). D, Epithelial components intermixed with the spindle cells in the tumor stroma (arrows) (H\&E stain, 400X). E, Epithelial component being highlighted by CK. F, Tumour cells showed strong and diffuse positivity with TLE-1 immunostains. 


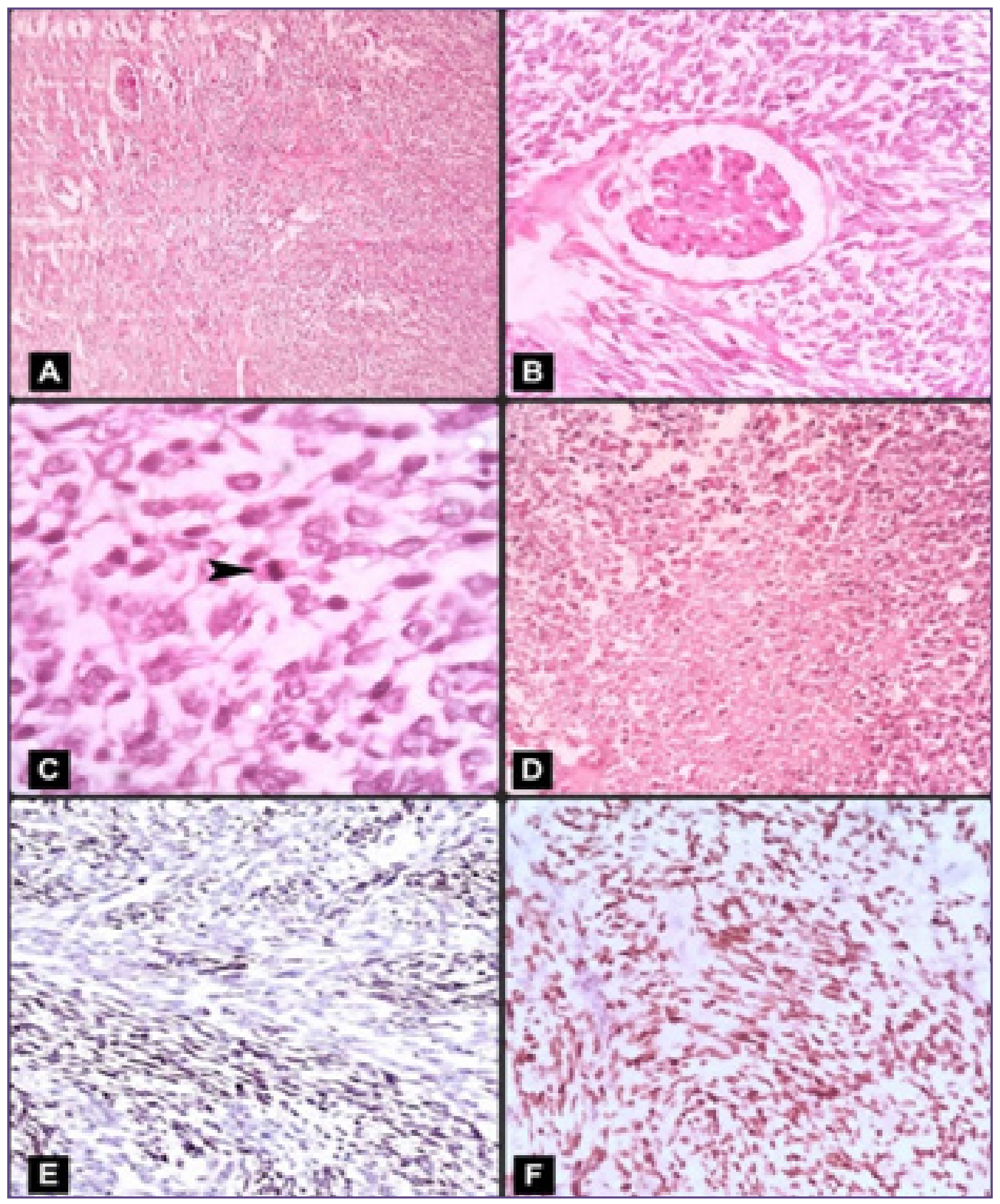

Fig. 3: (Case 3) A,Scanner magnification revealing a spindle cell tumor infiltrating the adjacent normal renal parenchyma (H\&E stain, 40X). B, Low magnification shows tumour cells around the glomerulus (H\&E stain, 100X)C, Occasional mitotic figures noted (H\&E stain, 400X). D, Few areas of necrosis were also seen (H\&E stain, 400X).E, Tumour cells showed strong and diffuse positivity with Bcl-2 and F, TLE-1 immunostains. 


\section{Discussion}

Synovial sarcoma is a relatively rare, malignant mesenchymal tumour showing partial epithelial differentiation. This tumour commonly arises in extremities, especially lower extremity and around the knee joint. However, it has been described in almost all other anatomical sites including rarely in the skin.[8] Synovial sarcoma occurs in older children, adolescents and young adults. Although it has been described in adults up to eighth and ninth decades, the peak incidence is in the third decade with $90 \%$ of cases occurring before 60 years of age. In a series of 271 patients by Ferrari et al, the median age at presentation was 32 years. The extremities were the most commonly involved site with 232 cases, followed by trunk (17 cases), head and neck (10 cases), abdominopelvic region ( 8 cases), mediastinum ( 2 cases) and lung ( 2 cases). [8] In another case series of 104 patients, a study done by the Scandinavian Sarcoma Group, the median age of presentation was 38 years. Location wise, 69 cases were involving the lower extremity, 26 cases involved the upper extremity and 09 cases involved the trunk region.[9] Apart from these, a few other case series showed similar age wise and location wise involvement by synovial sarcoma with the majority of the cases occurring in young adults with male preponderance and affecting the extremities more frequently.[5] Our cases occurred predominantly in young adults with two cases occurring in the age group of more than 55 years. However, we encountered cases in unusual sites such as the lung, heart and kidney which is an atypical occurrence in itself. [5,8,9] Synovial sarcoma arising primarily in the heart is a very rare occurrence, with only 24 previously reported cases. In the heart, they have been reported predominantly on the right sideparticularly the right atrium, with a right to the left ratio of 2:1 as mentioned in the largest series of cardiac synovial sarcomas reported by Kim et al in their 10-year singleinstitution report.[10] Our case also had an origin in the right atrium with extension into the right ventricle.

Primary pulmonary synovial sarcoma is an extremely rare tumour, accounting for less than $0.5 \% \cdot{ }^{[11]}$ Diagnosis of this entity can be made only after ruling out metastatic sarcomas and other primary lung tumours such as spindle cell variant of carcinomas

Histologically, synovial sarcoma is primarily of two subtypes: monophasic spindle cell and biphasic. Rarer subtypes include monophasic epithelial, round cell, calcifying, myxoid, and poorly differentiated type. Out of these, monophasic spindle cell is the commonest subtype comprising of hypercellular arrays of uniform spindle cells with scant amphophilic cytoplasm; ovoid, short or elongated vesicular nuclei with evenly dispersed chromatin and inconspicuous nucleoli. Usually, nuclear overlapping is seen with sparse stroma. Other characteristic findings include hemangiopericytomatous vascular pattern, mast cells, focal calcification with or without ossification.

Although histomorphology of synovial sarcoma is characteristic, a number of differentials need to be considered before giving out a definite diagnosis. ${ }^{[12,13]}$ Ancillary diagnostic methods like IHC, cytogenetics and fluorescence in situ hybridization (FISH) may be required to solve the diagnostic dilemma. Monophasic subtype is required to be differentiated from fibrosarcoma, malignant peripheral nerve sheath tumour, dedifferentiated liposarcoma, leiomyosarcoma and solitary fibrous tumour. ${ }^{[4]}$ Hence, a judicious use of IHC panel should be used keeping in mind all the differentials when approaching a case of soft tissue sarcoma, including BCL2, CD99 and TLE-1 whenever available.

A more definitive diagnostic approach to synovial sarcoma is the demonstration of translocation $\mathrm{t}(\mathrm{X}: 18)$ leading to SYT-SSX-1/SYT-SSX-4 fusion gene. ${ }^{[14]}$ More uncommon and rare translocations are the $\mathrm{t}(\mathrm{X}: 15)$ and $\mathrm{t}(\mathrm{X}: 7) \cdot{ }^{[15]}$

\section{Conclusion}

In our case series, we included cases that presented with tumours at different locations, which are rare sites for synovial sarcoma and had all the three histomorphological spectra. Differential diagnosis of synovial sarcoma should always be kept in mind in a young patient with a mass lesion of short duration, not only at the extremities but also involving other rarer locations like the lungs, heart and kidney.

\section{References}

1. Doyle LA. Sarcoma classification: An update based on the 2013 World Health Organization Classification of Tumors of Soft Tissue and Bone. Cancer. 2014;120(12):1763-74.

2. Chandrashekhar A. Sohoni. Synovial sarcoma. Appl Radiol. 2013;42(11):42-4.

3. Arun PS, Saravanan VAS, Aishwarya S. Synovial SarcomaA Review. IJCMR. 2017;4(6):1344-6.

4. Ruggiero A. Synovial sarcoma. Orphanet Encycl. 2004 Mar;1-5.

5. Cadman NL, Soule EH, Kelly PJ. Synovial sarcoma. An Analysis of 134 Tumors. Cancer. 1965 May 1;18(5):613-27.

6. Rekhi B, Basak R, Desai SB, Jambhekar NA. Immunohistochemical validation of TLE1, a novel marker for synovial sarcomas. Indian J Med Res. 2012 Nov;136(5):766-75.

7. Terry J, Saito T, Subramanian S, Ruttan C, Antonescu CR, Goldblum JR, et al. TLE1 as a Diagnostic 
Immunohistochemical Marker for Synovial Sarcoma Emerging From Gene Expression Profiling Studies. Am J Surg Pathol. 2007 Feb;31(2):240-6.

8. Ferrari A, Gronchi A, Casanova M, Meazza C, Gandola L, Collini P, et al. Synovial sarcoma: A retrospective analysis of 271 patients of all ages treated at a single institution. Cancer. 2004;101(3):627-34.

9. Skytting B. Synovial sarcoma. A Scandinavian Sarcoma Group project. Acta Orthop Scand Suppl. 2000;291(August):1-28.

10. Kim CH, Dancer JY, Coffee D, Zhai QJ, Reardon M, Ayala AG, et al. Clinicopathologic study of 24 patients with primary cardiac sarcomas: a 10-year single institution experience. Hum Pathol. 2008;39(6):933-938.

11. Mankin HJ, Hornicek FJ. Diagnosis, classification, and management of soft tissue sarcomas. Cancer Control. 2005;12:5-21.

12. Goldblum JR. An approach to pleomorphic sarcomas: can we subclassify, and does it matter? Mod Pathol. 2014 Jan 1;27:39-46.

13. Thway K, Fisher C. Synovial sarcoma: Defining features and diagnostic evolution. Ann Diagn Pathol. 2014 Dec;18(6):369-80.
14. Ladanyi M. Fusions of the SYT and SSX genes in synovial sarcoma. Oncogene. 2001 Sep 10;20(40):5755-62.

15. Limon J, Mrozek K, Mandahl N, Nedoszytko B, Verhest A, Rys J, et al. Cytogenetics of synovial sarcoma: presentation of ten new cases and review of the literature. Genes Chromosomes Cancer. 1991 Sep;3(5):338-45.

16. Cabuk D, Ustuner B, Akgul AG, Acikgoz O, Yaprak B, Uygun K, et al. Primary synovial sarcoma of lung. Korean J Thorac Cardiovasc Surg. 2014;47(3):306-9.

17. Report C. Primary Synovial Sarcoma of the Lung. Radiol Case Reports . 2006;2(4):679-83.

18. Boulmay B, Cooper G, Reith JD, Marsh R. Primary cardiac synovial sarcoma: A case report and brief review of the literature. Sarcoma. 2007;2007:10-3.

19. Dassi V, Das K, Singh BP, Swain SK. Primary synovial sarcoma of kidney: A rare tumor with an atypical presentation. Indian J Urol . 2009;25(2):269-71.

20. Modi G, Madabhavi I, Panchal H, Anand A, Patel A, Parikh S, et al. Primary synovial sarcoma of kidney: a rare differential diagnosis of renomegaly. Case Rep Pathol. 2014;2014(Figure 1):657497.

*Corresponding author:

Surg CdrDivya Shelly, Assistant Professor, Department of Pathology, Armed Forces Medical College, Sholapur Road, Pune-411040 India Phone: +91 9930676695

Email: dshelly13@gmail.com

Financial or other Competing Interests: None. 